\title{
Established and Validated Novel Nomogram for Predicting Prognosis of Post-Mastectomy pNO-I Breast Cancer without Adjuvant Radiotherapy
}

\author{
Wei-Xiang Qi \\ Lu Cao \\ Cheng Xu \\ Shengguang Zhao \\ Jiayi Chen
}

Department of Radiation Oncology, Ruijin Hospital, Shanghai Jiaotong

University School of Medicine, Shanghai, People's Republic of China
Correspondence: Jiayi Chen; Wei-xiang Qi Department of Radiation Oncology, Ruijin Hospital, Shanghai Jiaotong University School of Medicine, Shanghai, People's Republic of China

Email jiayichen2019@126.com; qiweixiangIII3@I63.com
Aim: To establish and validate a nomogram for predicting prognosis of breast cancer patients with pN0-1 who were treated with mastectomy and without adjuvant radiotherapy. Material and Methods: The LASSO regression was performed to identify predictors of breast cancer-specific survival (BCSS), local regional recurrence (LRR) and distant metastasis (DM). Model performance was evaluated by the concordance index (C-index) and calibration plot.

Results: The 5-year BCSS, LRR and DM rates for the entire cohort were $98 \%, 2 \%$ and $4 \%$, respectively. LASSO regression analysis found that pathological $\mathrm{T}$ stage, number of positive LN, grade and Ki-67 were significant predictors for both BCSS and DM-free survival, while number of resected LN and PR status were predictors for DM-free survival. In addition, number of positive LN was the only significant predictor for developing LRR. The C-indexes for the 5-year BCSS and DM nomograms were 0.81 and 0.78 in the training data set, 0.65 and 0.70 in the testing set and 0.72 and 0.69 in the external validation set, respectively.

Conclusion: Our prognostic nomograms accurately predict 5-year BCSS and DM-free survival in post-mastectomy breast cancer without adjuvant radiotherapy, which provides a useful tool to identify high-risk patients who could benefit from additional adjuvant therapy.

Keywords: breast cancer, pN0-1, mastectomy, LASSO analysis, nomogram, prognosis

\section{Introduction}

According to the report of GLOBOCAN 2018, female breast cancer remains the most frequent cancer among women worldwide, with 2.1 million newly diagnosed, which accounts for almost 1 in 4 cancer cases among women. ${ }^{1}$ In China, breast cancer is also the most common cancer diagnosed among women, with an upward stable trend in both incidence and mortality. ${ }^{2}$ During the past decades, the prognosis of early-stage breast cancer has been remarkably improved due to the great advances in cancer treatments. ${ }^{3,4}$ However, the optimal adjuvant treatment for this patient population ( $\mathrm{pN} 0-1)$ remains to be clearly determined. ${ }^{5}$

Recently, two large phase III randomized controlled trials, National Cancer Institute of Canada Clinical Trials Group MA20 ${ }^{6}$ and the European Organization for Research and Treatment of Cancer (EORTC) 22,922-10,925, ${ }^{7}$ reported the 10 year follow-up results, and both of them indicated that there was an improved disease-free survival with the addition of regional lymph node irradiation to wholebreast RT for early-stage breast cancers, which suggested that adjuvant radiotherapy 
in early-stage breast cancer not only decreased the risk of local regional recurrence (LRR), but also the risk of developing distant metastasis (DM). However, only $24 \%$ of patients in the EORTC trial were treated with mastectomy, and all of enrolled patients in MA20 were treated with lumpectomy. Consistently with these two trials, a large individual patient data meta-analysis of 8135 patients from 22 randomized trials showed that post-mastectomy radiotherapy (PMRT) could reduce locoregional recurrence $(p<0.001)$ and breast cancer mortality ( $\mathrm{RR} 0.80$, $p=0.01$ ) among BC patients with axillary dissection and one to three positive nodes. ${ }^{8}$

However, early-stage breast cancer is a heterogeneous disease; it has been reported that the LRR rates could increase to $20 \%$ in patients with multiple risk factors, including young age, large tumor size, lymph vascular invasion, medial/central tumor location or high nuclear grade. ${ }^{9,10}$ In addition, in a recent retrospective study published by Muhsen et al, the authors found that nearly $85 \%$ of patients with T1-2N1 could be spared PMRT due to the low incidence of LRR. ${ }^{11}$ As a result, the role of adjuvant PMRT in early-stage breast cancer remains controversial, and an accurate prognostic indicator to identify patients who are at high risk for recurrence is clearly needed by physicians in their individualized clinical decision making. Nomograms are frequently used not only for predicting survival in patients with all types of cancer but also for successfully quantifying risk prediction according to clinicopathological variables. ${ }^{12-15}$ Although many of the available decision tools, such as CancerMath, ${ }^{16}$ PREDICT $^{17}$ and the ipsilateral breast tumor recurrence program (IBTR), ${ }^{18,19}$ have been established to predict survival probability in breast cancer patients, none of these tools are established specially based on the post-mastectomy breast cancer with pN0-1 without adjuvant radiotherapy. As a result, we perform the present study to establish and validate a nomogram based on a large sample size for predicting prognosis of post-mastectomy breast cancer with pN0-1, but without adjuvant radiotherapy, which could be used for adjuvant treatment counseling after mastectomy.

\section{Materials and Methods}

\section{Study Cohorts and Data Collection}

Between Jan 2009 and Dec 2015, a total of 2128 newly diagnosed invasive breast cancer patients undergoing a modified radical mastectomy or total mastectomy and sentinel lymph node biopsy with pathological (p) N0-1, who were not treated with adjuvant radiotherapy, were identified at our institution. Two hundred and forty-nine patients were excluded from the present analysis for the following reasons: (1) neoadjuvant chemotherapy or radiotherapy; (2) bilateral breast cancers; (3) lack of information about tumor size, Ki-67 and hormonal receptor status; (4) the pathologic diagnosis was ductal carcinoma in situ (DCIS) or Paget's disease. Patients with positive surgical margin were also excluded for analysis in the present study. Finally, the remaining 1879 patients were included for analysis in the present study (Figure 1). The present study procedures were approved by the Ethical Committee of RuiJin Hospital affiliated medicine school of Shanghai Jiao Tong University [2020 (250)].

Among a total of 3871 patients from one phase III trial (NCT00041119), ${ }^{20}$ which aimed to compare the efficacy difference of adjuvant chemotherapy of doxorubicin and cyclophosphamide versus single-agent paclitaxel for breast cancer in women with pN0-1, 1359 patients treated with mastectomy were included. Subsequently, 3 patients with more than 3 positive lymph node metastases were excluded. Finally, a total of 1356 patients were used as an externally validated cohort in the present study. The primary findings of this trial had been previously published.

\section{Outcomes Definitions}

The primary endpoints of the current analysis were breast cancer-specific survival (BCSS), which is defined as the time from surgery till death from breast cancer. Local regional recurrence (LRR)-free survival was defined as the time from surgery to the time of a first recurrence in the ipsilateral breast or in axillary, supraclavicular or internal mammary nodes. Distant metastasis (DM)-free survival was defined as the time from surgery to the time of a recurrence at a distant site.

\section{Statistical Analysis}

Statistical analyses were conducted through $\mathrm{R}$ version 3.6.1 software (The $\mathrm{R}$ Foundation for Statistical Computing, Vienna, Austria; http://www.r-project.org) and NCSS 11.0 software. Continuous variables were summarized by median and range, and categorical variables were summarized by frequency and proportion. The study population was randomly dichotomized into 2 groups: $70 \%$ in the training (1315) and 30\% (564) in the testing group, respectively. Comparisons of baseline characteristics between training and testing groups were performed with $\chi$ test. Time-dependent variables were assessed through Kaplan-Meier analysis. 


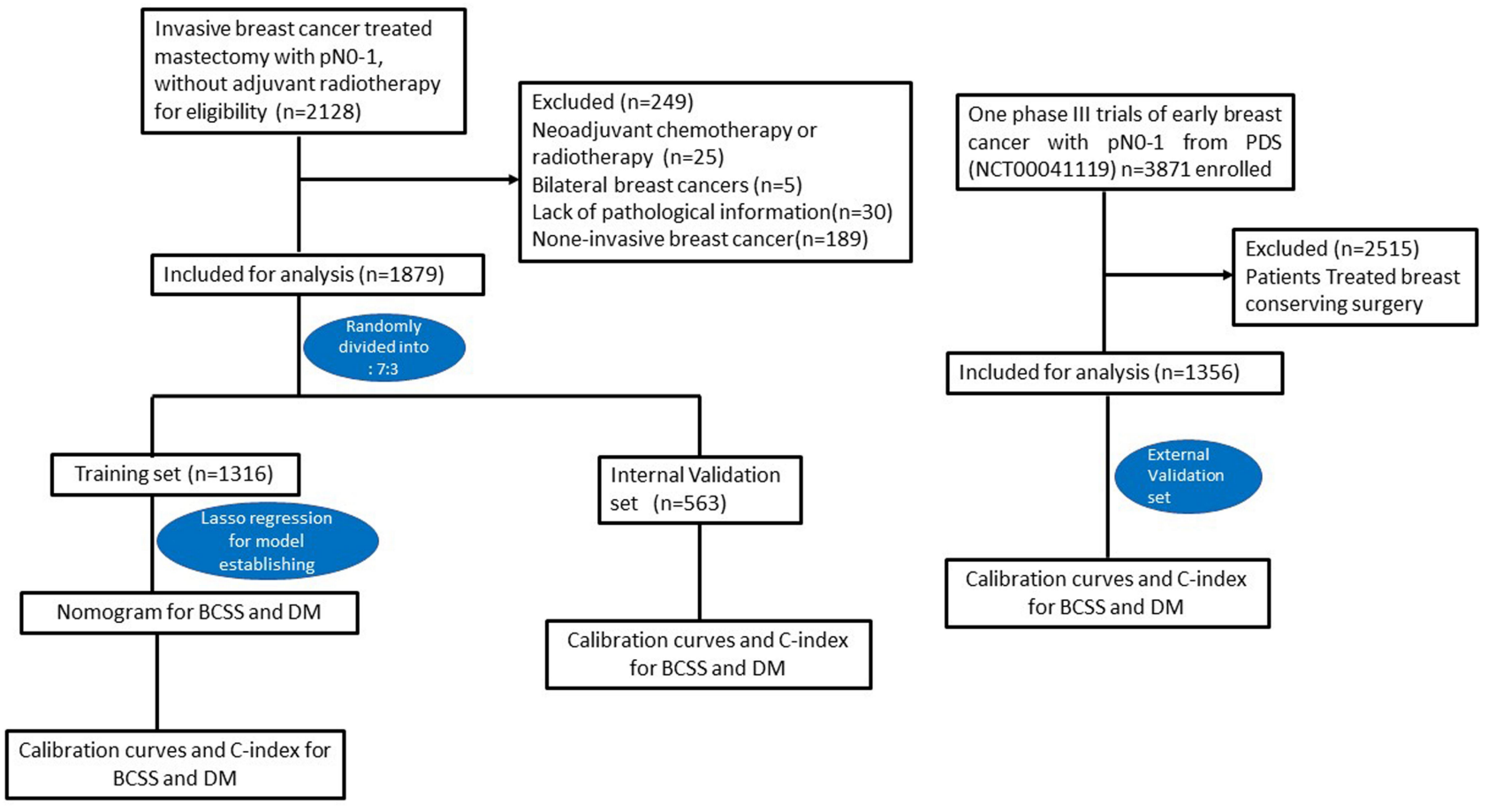

Figure I Flow of patient selection and external validation.

\section{Nomogram Development}

The training data set $(n=1315)$ was used for initial nomogram development. The least absolute shrinkage and selection operator (LASSO) was employed for variable selection to refine the model structures for BCSS, DM-free survival and LRR-free survival, with the optimal LASSO penalty determined using a 10-fold cross-validation. The variables used in the analysis were age $(20-29,30-39,40-49,50-59,60-69$ or $\geq 70$ years), Charlson Comorbidity Index (CCI) score, Ki-67, ER status (positive or negative), PR status (positive or negative), HER-2 status (positive or negative), grade (grade 1, grade 2 or grade 3), pathological T stage (pT1, pT2 and pT3), number of positive lymph nodes (LN), number of resected $\mathrm{LN}$, positive $\mathrm{LN}$ ratio, tumor location (medial or lateral), molecular types of breast cancer (luminal A, luminal B, HER-2 overexpression and triple-negative breast cancer) and adjuvant systematic therapy including chemotherapy (yes or no), endocrine therapy (yes or no) and anti-HER-2 therapy (yes or no). The breast cancer subtypes were classified based on the St Gallen consensus 2013, ${ }^{21}$ luminal A type was defined as ER and PR positive, HER-2 negative and Ki- $67<14 \%$; luminal B type (HER-2 negative) was defined as ER positive, HER-2 negative, PR negative and/or Ki- $67 \geq 14 \%$; luminal B type (HER-2 positive) was defined as ER positive, HER-2 positive, any Ki-67 and any PR status; HER-2 positive was defined as HER-2 over-expression or amplified, ER and PR negative; triple-negative was defined as ER negative, PR negative and HER-2 negative. A nomogram for possible prognostic factors associated with BCSS, and DM-free survival was established by R software.

\section{Nomogram Validation}

Calibration curves were plotted to assess the agreement between the actual rate of BCSS and DM-free survival and the predicted probabilities of BCSS and DM-free survival. To obtain a relatively unbiased estimate, bootstrapping method was used with 1000 re-samplings to produce the calibration plot. A calibration curve of $45^{\circ}$ indicates a perfect prognostic prediction. In addition, the predictive accuracy was evaluated by Harrell's concordance index (C-index), which ranges from 0.5 (random chance) to 1 (perfect prediction). If the $\mathrm{C}$-index exceeds 0.7 , the model is generally considered as good, and excellent if the $\mathrm{C}$-index is more than 0.8 . Finally, the prediction model was validated using the external data set from one phase III trial mentioned above.

\section{Results}

\section{Baseline Characteristics}

From Jan 2009 to Dec 2015, a total of 1879 consecutive patients with breast cancer with $\mathrm{pN} 0-1$ who were treated with 
mastectomy were included for analysis. Firstly, we randomly divided them into a training cohort and a testing cohort (7:3), with 1316 patients in the training cohort and 563 in the validation cohort. No variables were significantly different between the two cohorts. The median age at diagnosis was 56 years (range, 28-92 years) in the training set and 58 years (range, 23-92 years) in the testing set. The median tumor size was $2 \mathrm{~cm}$ in both cohorts. The majority of our cohorts (1590 patients, $84.6 \%$ ) did not have lymph node (LN) metastasis. A total of 1731 patients $(92.1 \%)$ were treated with adjuvant systematic therapy, and 623 patients $(33 \%)$ were treated with both adjuvant chemotherapy and hormonal therapy. Adjuvant chemotherapy was received by 785 patients $(60 \%)$ in the training cohort and by 311 (55.2\%) in the validation cohort. Among 1096 patients who received chemotherapy, 537 patients (49\%) were treated with doxorubicin, cyclophosphamide and paclitaxel; 511 patients $(46.6 \%)$ were treated with doxorubicin-containing or taxanecontaining regimens, and the remaining 48 patients $(4.4 \%)$ were treated with other regimens. Adjuvant hormonal therapy was received by 868 patients $(66.0 \%)$ in the training cohort and by 383 patients $(68 \%)$ in the validation cohort. Among 1251 patients treated with hormonal therapy, 698 patients $(55.8 \%)$ were treated with aromatase inhibitors (AIs), and 549 patients (43.9\%) were treated with selective estrogen receptor modulators (SERMs). Detailed baseline characteristics are listed in Table 1.

\section{Survival Outcomes}

By the latest follow-up of Oct 2019, with a median 60 months of follow-up, a total of $101(5.37 \%)$ patients died in the entire cohort, with $55(2.93 \%)$ patient deaths attributed to breast cancer and the remaining $46(2.45 \%)$ deaths due to other reasons. The survival outcomes of the entire cohort were excellent. The 5- and 10-year breast cancer-specific survival (BCSS) was 98\% and 95\%, respectively (Supplemental figure $1 \mathrm{~A}$ and $\underline{\mathrm{B}}$ ). The 5- and 10-year overall survival (OS) was $97 \%$ and $91 \%$, respectively (Supplemental Figure 1C and D). A total of 44 patients developed local regional recurrences, with 5- and 10 -year cumulative LRR rates of $2 \%$ and $3 \%$ (Supplemental Figure 2), respectively. In addition, a total of 90 patients developed distant metastasis (DM), and the 5- and 10-year cumulative DM rates were $4 \%$ and $6 \%$, respectively (Supplemental Figure 2). In the external validation cohort, the 5-year OS and BCSS were 95\% and 97\%, respectively (Supplemental Figure 3), and the cumulative incidences of 5-year LRR and DM were 3\% and 6\%, respectively (Supplemental Figure 3).

\section{Factors Associated with BCSS, LRR and DM}

A total of 16 variables were considered as potential predictors. We used a LASSO regression algorithm based on each variable for predictor selection in the training cohort. As shown in Figure 2, when the partial likelihood deviance reached its minimum value the appropriate tuning parameter $\gamma$ was 0.0059 and $\log \gamma$ was -5.14 ; five variables with nonzero coefficients were obtained from the LASSO analysis.

As for LRR-free survival, LASSO regression showed that only the number of positive LN was the significant predictor. And univariate Cox analysis also found that number of positive $\mathrm{LN}$ was a significant risk factor for LRR (HR 1.71, 95\% CI: 1.13-2.60, $p=0.027$, Table 2); additionally, ten variables with nonzero coefficients, including tumor location, age, number of positive LN, pathological T stage, Ki-67, PR status, grade, number of resected $\mathrm{LN}$, adjuvant hormonal therapy and anti-HER-2 therapy, were obtained from the LASSO analysis.

\section{Construction of the Nomogram}

Univariate analysis showed that all of the five variables were significant predictors for BCSS (all $p<0.05$ ). Multivariate Cox regression analysis showed that the four variables of age $(p=0.052)$ number of positive $\mathrm{LN}$ $(p<0.001)$, pathological $\mathrm{T}$ stage $(p=0.021$ and $p<0.001)$ and Ki-67 ( $p=0.005$, Table 2 ) have independent prognostic significance for BCSS. All of these five variables were selected for the construction of nomogram of BCSS (Figure 3A). The newly developed predictive model showed good discrimination with a C-index of 0.81 . An excellent concordance between the predicted and observed 5-year BCSS probabilities was observed in the calibration plot (Figure 4).

Additionally, univariate analysis showed that six of the ten variables were significant predictors for DM-free survival (number of positive LN, pathological T stage, Ki-67, number of resected LN, grade and PR status, $p<0.05$ ); multivariate Cox regression analysis showed that four variables, number of positive $\mathrm{LN}(p=0.002)$, pathological $\mathrm{T}$ stage ( $p=0.01$ and $p=0.002), \mathrm{Ki}-67(p=0.018)$ and total of resected LN ( $p=0.037$ ), had independent prognostic significance for DM (Table 2). Finally, six variables were selected for the 
Table I Baseline Characteristics of Included Patients

\begin{tabular}{|c|c|c|c|}
\hline Group & Training Set $(1316)$ & Validation Set $(n=563)$ & $P$ value \\
\hline Age (median, range) & $56(28-92)$ years & $58(23-92)$ years & \\
\hline \multicolumn{4}{|l|}{$\mathrm{N}$ stage } \\
\hline pNo & 1115 & 475 & 0.90 \\
\hline $\mathrm{pNI}$ & 201 & 88 & \\
\hline $\mathrm{CCl}$ score & I (0-8) & $2(0-7)$ & \\
\hline \multicolumn{4}{|l|}{ Location } \\
\hline Medial & 409 & 191 & 0.25 \\
\hline Lateral & 907 & 372 & \\
\hline T size (median, range) & $2(0.01-14) \mathrm{cm}$ & $2(0.02-12.5) \mathrm{cm}$ & \\
\hline \multicolumn{4}{|l|}{ T stage } \\
\hline TImic & 36 & 11 & 0.65 \\
\hline Tla & 77 & 26 & \\
\hline TIb & 172 & 72 & \\
\hline TIc & 521 & 221 & \\
\hline $\mathrm{T} 2$ & 484 & 224 & \\
\hline T3 & 26 & 9 & \\
\hline \multicolumn{4}{|l|}{ Grade } \\
\hline 1 & 119 & 58 & 0.57 \\
\hline 2 & 742 & 321 & \\
\hline 3 & 455 & 184 & \\
\hline \multicolumn{4}{|l|}{ Adjuvant chemotherapy } \\
\hline Yes & 790 & 313 & 0.08 \\
\hline No & 526 & 250 & \\
\hline \multicolumn{4}{|c|}{ Adjuvant anti-HER-2 therapy } \\
\hline Yes & $17 \mid$ & 70 & 0.80 \\
\hline No & 1145 & 493 & \\
\hline \multicolumn{4}{|l|}{ Number of positive LN } \\
\hline 0 & 1115 & 475 & 0.28 \\
\hline 1 & 133 & 56 & \\
\hline 2 & 49 & 17 & \\
\hline 3 & 19 & 15 & \\
\hline Number of resected LN & $13(1-46)$ & $13(1-38)$ & \\
\hline \multicolumn{4}{|l|}{ ER status } \\
\hline Positive & 857 & 376 & 0.52 \\
\hline Negative & 459 & 187 & \\
\hline \multicolumn{4}{|l|}{ PR status } \\
\hline Positive & 539 & 235 & 0.79 \\
\hline Negative & 777 & 328 & \\
\hline \multicolumn{4}{|l|}{ HER-2 status } \\
\hline Positive & 294 & 123 & 0.79 \\
\hline Negative & 1022 & 440 & \\
\hline
\end{tabular}


Table I (Continued).

\begin{tabular}{|c|c|c|c|}
\hline Group & Training Set $(1316)$ & Validation Set $(n=563)$ & $P$ value \\
\hline \multicolumn{4}{|l|}{ Adjuvant hormonal therapy } \\
\hline Yes & 868 & 383 & 0.41 \\
\hline No & 448 & 180 & \\
\hline \multicolumn{4}{|l|}{ Sub-groups } \\
\hline ER positive/HER-2 negative & 743 & 329 & 0.86 \\
\hline ER negative/HER-2 negative & 279 & 111 & \\
\hline ER positive/HER-2 positive & 114 & 47 & \\
\hline ER negative/HER-2 positive & 180 & 76 & \\
\hline \multicolumn{4}{|l|}{ Molecular types } \\
\hline Luminal A & 457 & 186 & 0.60 \\
\hline Luminal B & 413 & 194 & \\
\hline HER-2 positive & 178 & 76 & \\
\hline Triple-negative & 268 & 107 & \\
\hline
\end{tabular}

construction of a nomogram of DM-free survival (Figure 3B). The C-index of the nomogram in the testing data set was 0.77 , and the calibration plot indicated that there was a good concordance between the predicted and observed 5-year DM-free survival probabilities (Figure 4).

\section{Internal Validation of Nomogram}

In the internal validation data set of 563 patients, the C-index of the internal test data set for BCSS and DM-free survival was 0.65 and 0.70 , respectively. The calibration plot was used to compare the difference between predicted

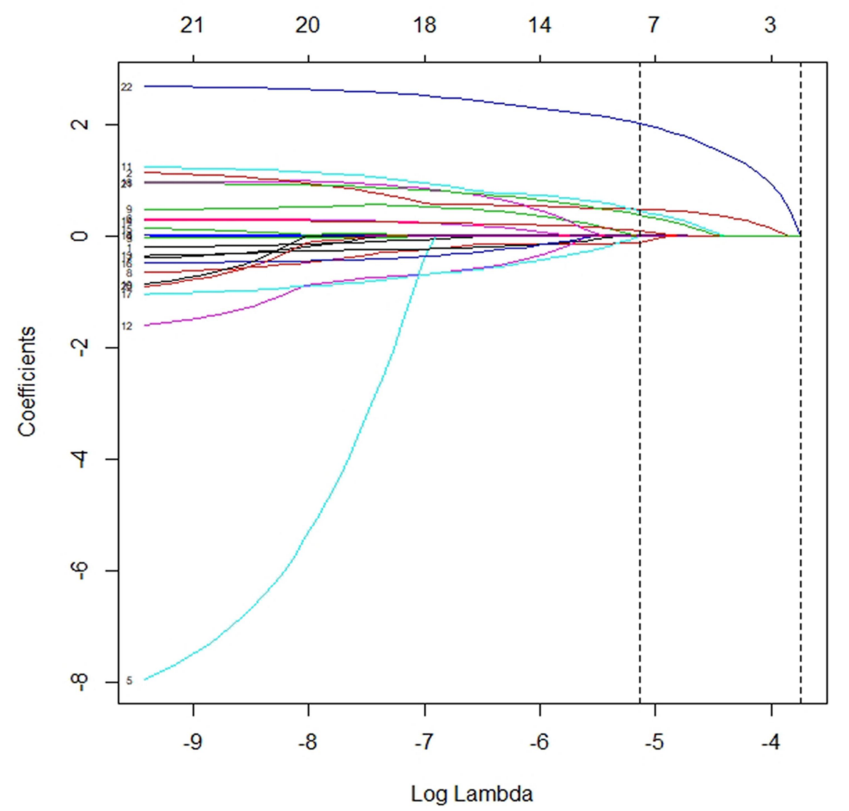

5-year BCSS and DM-free survival probabilities and the actual 5-year survival probabilities. Our result showed that the calibration curve revealed good concordance between the predicted and observed probabilities (Figure 4).

\section{External Validation of Nomogram}

The model was externally validated in an independent cohort of 1356 breast cancer patients from one phase III trial (NCT00041119). As the Ki-67 data could not be obtained from the phase III trial, we constructed a modified nomogram based on four variables including

$\begin{array}{lllllllllllllllll}22 & 21 & 21 & 21 & 20 & 20 & 18 & 16 & 16 & 14 & 13 & 9 & 7 & 5 & 5 & 3 & 2\end{array}$

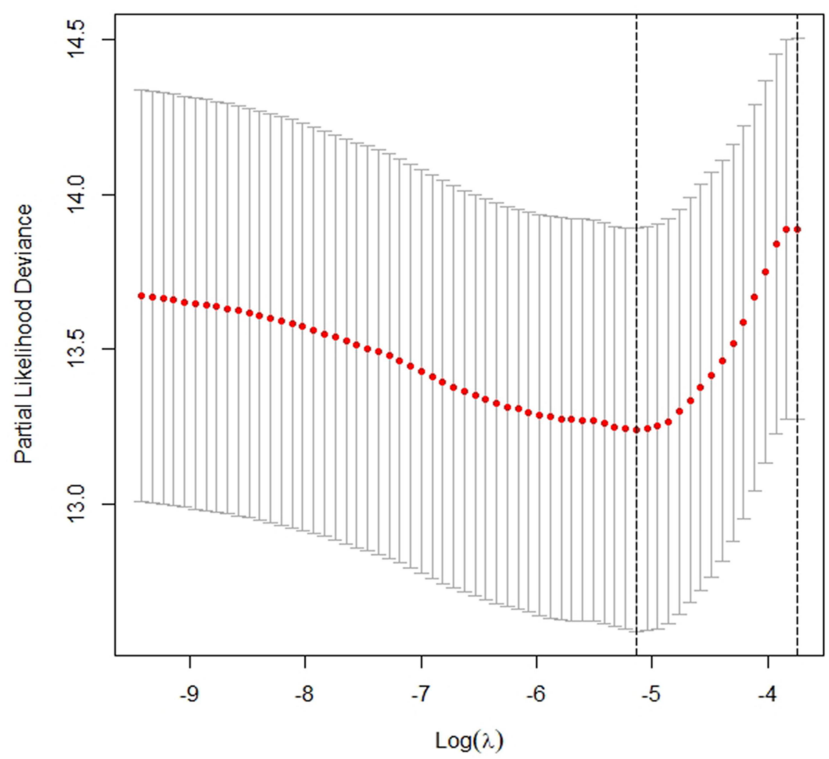

Figure 2 Feature selection using least absolute shrinkage and selection operator (LASSO) Cox regression for BCSS. 
Table 2 Multi-Variable Cox Regression of Selected Variables in the Nomogram

\begin{tabular}{|c|c|c|c|}
\hline \multirow[t]{2}{*}{ Group } & BCSS & LRR & DM \\
\hline & HR $(95 \% \mathrm{Cl}), p$ value & HR $(95 \% \mathrm{Cl}), p$ value & HR (95\% CI), $p$ value \\
\hline Age & $1.03(1.00-1.05), p=0.052$ & - & - \\
\hline Number of positive LN & I.77 (I.29-2.46), $p<0.00 \mathrm{I}$ & I.7I $(1.13-2.60), p=0.027$ & 1.55 (I.I7-2.06), $p=0.002$ \\
\hline \multicolumn{4}{|l|}{ T stage } \\
\hline $\mathrm{pTl}$ & Reference & - & Reference \\
\hline pT2 & 2.25 (I.I3-4.52), $p=0.021$ & - & $1.98(1.18-3.32), p=0.01$ \\
\hline pT3 & | 3.04 (4.6|-36.88), $p<0.00$ | & - & $4.99(1.77-14.06), p=0.002$ \\
\hline \multicolumn{4}{|l|}{ Grade } \\
\hline $\mathrm{I}-2$ & Reference & - & Reference \\
\hline 3 & $1.38(0.68-2.79), p=0.37$ & - & I.I7 (0.68-2.04), $p=0.55$ \\
\hline Ki-67 & $1.02(1.00-1.033), p=0.005$ & - & $1.01(1.00-1.02), p=0.020$ \\
\hline Number of resected LN & - & - & $1.04(1.00-1.07), p=0.039$ \\
\hline \multicolumn{4}{|l|}{ PR status } \\
\hline Positive & - & - & Reference \\
\hline Negative & - & - & $0.68(0.37-1.23), p=0.20$ \\
\hline
\end{tabular}

age, number of positive $\mathrm{LN}$, pathological $\mathrm{T}$ stage and grade. The C-index for the modified model for BCSS in the training and external validation data set was 0.79 and 0.72 , respectively. The calibration plot also revealed good concordance between the predicted and observed probabilities in the external data set (Figure 4).

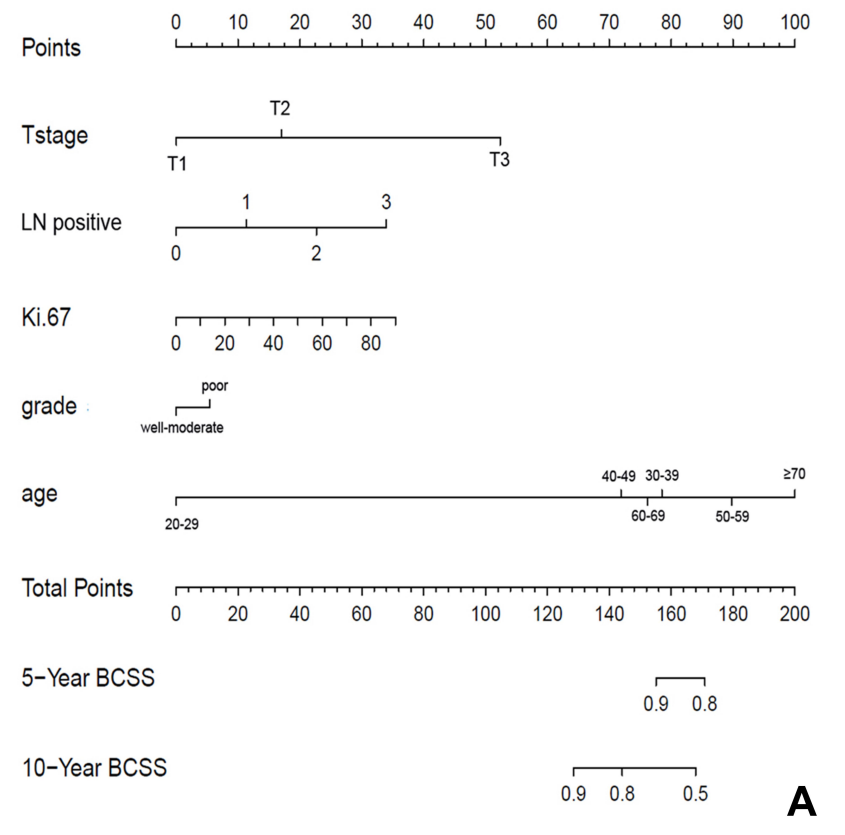

In addition, Ki-67 and total resected $\mathrm{LN}$ could not be obtained from the trial, thus a modified nomogram for DM-free survival based on the four variables pathological T stage, number of positive LN, grade and PR status was established in the external validation cohort. The C-index for the modified model for DM-free survival in the

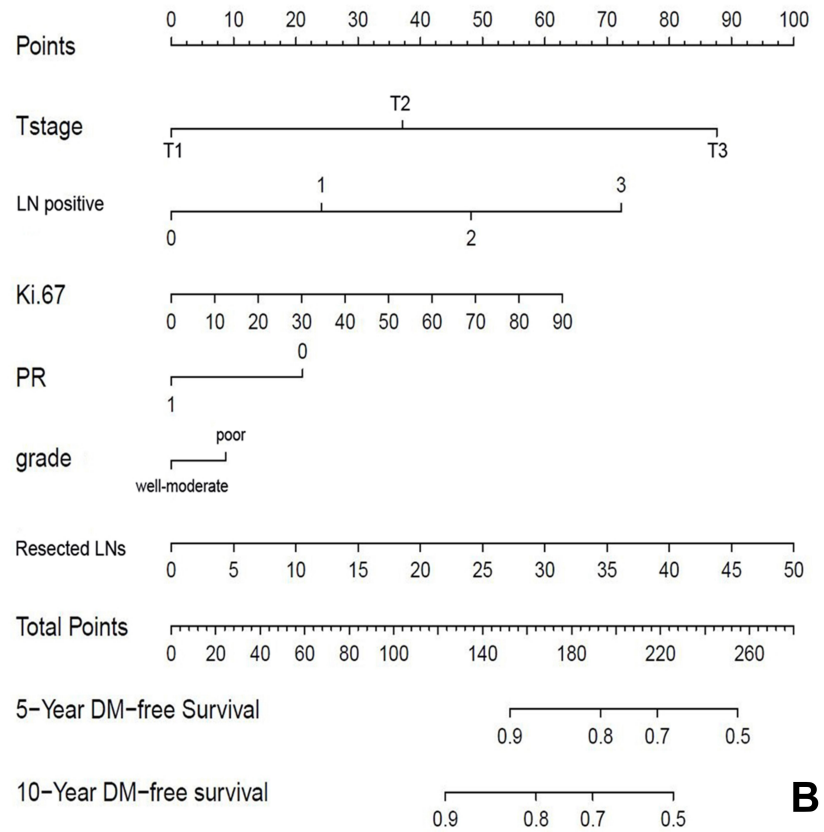

Figure 3 Nomogram for predicting 5-year BCSS (A) and 5-year DM-free survival (B). 

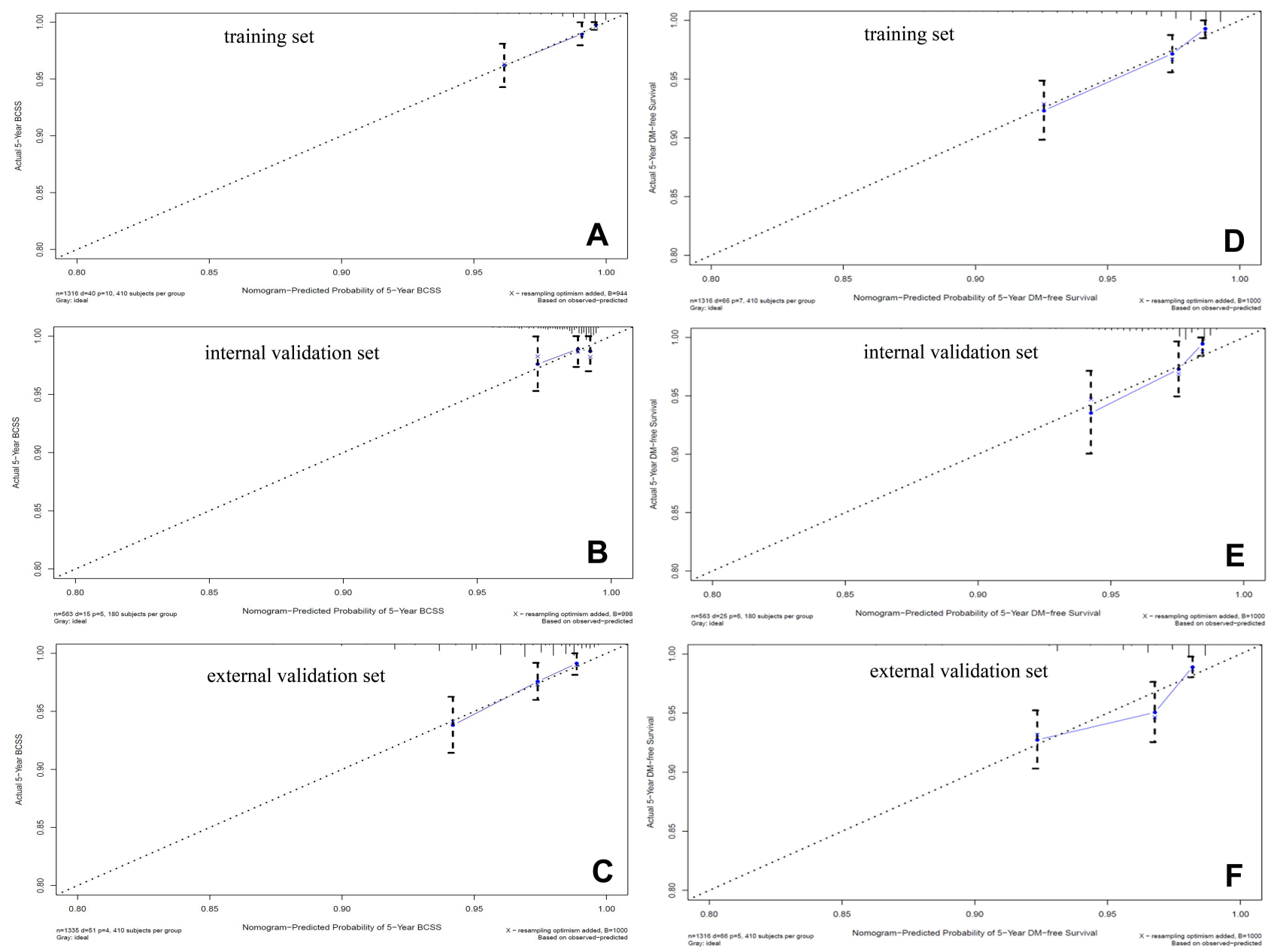

Figure 4 Calibration curves for 5-year BCSS and 5-year DM-free survival in the training, internal validation and external validation cohort: (A) the predicted and observed 5-BCSS in training set; (B) the predicted and observed 5-BCSS in internal validation set; (C) the predicted and observed 5-BCSS in external validation set; (D) the predicted and observed 5-year DM-free survival in training set; (E) the predicted and observed 5-year DM-free survival in internal validation set; (F) the predicted and observed 5-year DM-free survival in external validation set.

training and external validation data set was 0.72 and 0.69 , respectively. A good concordance between the predicted and observed 5-year DM-free survival probabilities in the external data set was observed (Figure 4).

\section{Discussion}

To our best knowledge, this is the first paper to establish and externally validate a novel nomogram to predict individualized 5-year BCSS and DM-free survival for early-stage postmastectomy breast cancer with pN0-1, but without adjuvant radiotherapy. In the present study, a total of 1879 postmastectomy breast cancer patients with pN0-1 without adjuvant radiotherapy from our single institute were enrolled. In our patient population, $84.6 \%$ present with lymph node negative disease, and $58.7 \%$ of the patients receives adjuvant chemotherapy. Initially, a total of 16 variables, including age,
CCI score, Ki-67, ER status, PR status, HER-2 status, grade, pathological T stage, number of positive lymph nodes (LN), number of resected $\mathrm{LN}$, positive $\mathrm{LN}$ ratio, tumor location (medial or lateral), molecular types of breast cancer, and adjuvant systematic therapy including chemotherapy (yes or no), endocrine therapy (yes or no) and anti-HER2 therapy (yes or no), are identified for LASSO regression analysis, which effectively processes the demographic and clinical feature selection as a statistical method for highdimensional data. ${ }^{22,23}$ Finally, five variables, including age, grade, pathological $\mathrm{T}$ stage, number of positive lymph node (LN) and Ki-67 with nonzero coefficients were distinguished for predictors of BCSS, one risk factor of number of positive LN for developing LRR, and six of ten variables with nonzero coefficients, including number of positive LN, pathological T stage, Ki-67, PR status, grade and number of resected 
$\mathrm{LN}$, were predictors for DM. We included these risk factors in the construction of nomograms for BCSS and DM-free survival, and both the $\mathrm{C}$-index values and the calibration diagrams showed satisfactory robustness when applied to both internal and external validation cohorts.

Prior to the present study, several retrospective studies have been published to investigate the risk factors for developing LRR in early-stage breast cancer. ${ }^{24-27}$ Sharma et al found that young age was an independent risk factor for developing LRR in patients with pT1-2 with 0 to 3 positive LN in 1019 patients during 1997-2002. The overall 5- and 10year LRR rates were $1.6 \%$ and $2.7 \%{ }^{28}$ Then, Truong et al analyzed 1505 T1-2N0 breast cancer patients between 1989 and 1999, without adjuvant radiotherapy, and logistic regression analysis indicated that grade, lympho-vascular invasion (LVI), $\mathrm{T}$ stage and systematic therapy are predictors for LRR. $^{27}$ More recently, Mamtani et $\mathrm{al}^{9}$ performed a retrospective analysis of 657 patients with pT1-2N0 postmastectomy breast cancer treated with modern systematic therapy and found that only tumor size was a risk factor for developing LRR ( $p=0.006$ ), and the crude LRR rate in the overall population was $4.7 \%$. In our population, the crude LRR rate was $2.3 \%$, and the only risk factor for LRR was the number of positive LN, although tumor size had a tendency to increase the risk of developing LRR $(1.15,95 \%$ CI: $0.98-1.35, p=0.097)$. One possible reason for this finding is that the proportion of patients who received adjuvant systematic therapy in our population is higher than in Mamtani et al's study $(92.1 \%$ vs $86 \%)$. In the external validation cohort, the cumulative incidence of 5-year LRR and DM was 3\% and 6\%, respectively, which is comparable to our cohorts.

Since the publication of MA20 and EORTC study, more and more oncologists agree that adjuvant radiotherapy in early-stage breast cancer not only decreases the LRR risk, but also the risk of distant metastasis. As a result, we also investigate the predictors for BCSS and DM-free survival in this patient cohort. Our analysis finds that pathological T stage, number of positive LN, grade and $\mathrm{Ki}-67$ are significant predictors for both BCSS and DM-free survival in postmastectomy breast cancer with $\mathrm{pN} 0-1$, while number of resected LN and PR status are also predictors for DM-free survival. In our nomogram, $\mathrm{Ki}-67$ is identified as a risk factor for both BCSS and DM. This finding is supported by previous studies, which indicates Ki-67 overexpression as an independent predictor for poor prognosis. Matsubara et a ${ }^{29}$ retrospectively analyzed 1166 early-stage patients and found that Ki-67 overexpression (Ki-67 $\geq 10 \%$ ) was identified as an independent prognostic factor for distant-metastatic-free survival
(DFS) and overall survival (OS) by univariate and multivariate analysis. Regarding another four prognostic factors for BCSS, including age, pathological $\mathrm{T}$ stage, number of positive $\mathrm{LN}$ and grade, multiple studies have reported these three factors as predictors for survival.

There are some limitations to our study. First, LVI status has been reported as a risk for poor survival in previous studies, ${ }^{30}$ but LVI status was not reported in our institute until Jan 2012, and thus we could not assess the prognostic role of LVI status in this patient population. Second, our study is a retrospective study, and as inherent biases are unavoidable in any retrospective study, a large prospective investigation is still needed to confirm our results. Thirdly, although the nomogram models are validated by an external cohort of 1356 patients from a phase III trial, radiation information of these patients is unavailable, and we do not know the percentage of patients who received radiation therapy. Thus the external validation set could be potentially compromised by including radiated patients. Further studies externally validating the models in this patient population are recommended. Finally, applying these nomogram models in the setting of particularly large tumors $(>5 \mathrm{~cm})$ should be done cautiously, because of the limited number of such patients $(n=35)$ within our study population.

\section{Conclusion}

In summary, we established and validated a novel nomogram for predicting survival of early-stage post-mastectomy pN0-1 breast cancer patients without adjuvant radiotherapy. This nomogram could help physicians to accurately evaluate the individual BCSS and DM-free survival in this patient population, and distinguish potential high-risk patients who could benefit from additional treatment.

\section{Ethical Approval}

All procedures performed in studies involving human participants were in accordance with the ethical standards of the institutional and/or national research committee and with the 1964 Helsinki declaration and its later amendments or comparable ethical standards. The present study procedures were approved by the Ethical Committee of RuiJin Hospital affiliated medicine school of Shanghai Jiao Tong University (2020-250).

\section{Informed Consent}

The information is coded, and the patients are deidentified. Consent was not required to be obtained. 


\section{Author Contributions}

All authors made a significant contribution to the work reported, whether that is in the conception, study design, execution, acquisition of data, analysis and interpretation, or in all these areas; took part in drafting, revising or critically reviewing the article; gave final approval of the version to be published; have agreed on the journal to which the article has been submitted; and agree to be accountable for all aspects of the work.

\section{Funding}

This study was supported in part by Clinical Research Plan of SHDC (grant SHDC2020CR2052B and SHDC2020CR4070).

\section{Disclosure}

The authors declare that they have no conflicts of interest.

\section{References}

1. Bray F, Ferlay J, Soerjomataram I, Siegel RL, Torre LA, Jemal A. Global cancer statistics 2018: GLOBOCAN estimates of incidence and mortality worldwide for 36 cancers in 185 countries. CA Cancer J Clin. 2018;68(6):394-424. doi:10.3322/caac.21492

2. Chen W, Zheng R, Baade PD, et al. Cancer statistics in China, 2015. CA Cancer J Clin. 2016;66(2):115-132. doi:10.3322/caac.21338

3. Wormann B. Breast cancer: basics, screening, diagnostics and treatment. Med Monatsschr Pharm. 2017;40(2):55-64.

4. Ponde NF, Zardavas D, Piccart M. Progress in adjuvant systemic therapy for breast cancer. Nat Rev Clin Oncol. 2019;16(1):27-44. doi:10.1038/s41571-018-0089-9

5. Gregucci F, Fozza A, Falivene S, et al. Present clinical practice of breast cancer radiotherapy in Italy: a nationwide survey by the Italian Society of Radiotherapy and Clinical Oncology (AIRO) Breast Group. Radiol Med. 2020;125(7):674-682. doi:10.1007/s11547-02001147-5

6. Whelan TJ, Olivotto IA, Parulekar WR, et al. Regional nodal irradiation in early-stage breast cancer. $N$ Engl $J$ Med. 2015;373 (4):307-316. doi:10.1056/NEJMoa1415340

7. Poortmans PM, Collette S, Kirkove C, et al. Internal mammary and medial supraclavicular irradiation in breast cancer. $N$ Engl $J$ Med. 2015;373(4):317-327. doi:10.1056/NEJMoa1415369

8. Ebctcg MP, Taylor C, et al. Effect of radiotherapy after mastectomy and axillary surgery on 10-year recurrence and 20 -year breast cancer mortality: meta-analysis of individual patient data for 8135 women in 22 randomised trials. Lancet. 2014;383(9935):2127-2135. doi:10.1016/S0140-6736(14)60488-8

9. Mamtani A, Patil S, Stempel MM, Morrow M. Are there patients with $\mathrm{T} 1$ to $\mathrm{T} 2$, lymph node-negative breast cancer who are "highrisk" for locoregional disease recurrence? Cancer. 2017;123 (14):2626-2633. doi:10.1002/cncr.30658

10. Abi-Raad R, Boutrus R, Wang R, et al. Patterns and risk factors of locoregional recurrence in T1-T2 node negative breast cancer patients treated with mastectomy: implications for postmastectomy radiotherapy. Int J Radiat Oncol Biol Phys. 2011;81(3):e151-e157. doi:10.1016/j.ijrobp.2011.01.015
11. Muhsen S, Moo TA, Patil S, et al. Most breast cancer patients with T1-2 tumors and one to three positive lymph nodes do not need postmastectomy radiotherapy. Ann Surg Oncol. 2018;25 (7):1912-1920. doi:10.1245/s10434-018-6422-9

12. Valentini V, Van Stiphout RG, Lammering G, et al. Nomograms for predicting local recurrence, distant metastases, and overall survival for patients with locally advanced rectal cancer on the basis of European randomized clinical trials. $J$ Clin Oncol. 2011;29 (23):3163-3172. doi:10.1200/JCO.2010.33.1595

13. Stephenson AJ, Scardino PT, Eastham JA, et al. Postoperative nomogram predicting the 10-year probability of prostate cancer recurrence after radical prostatectomy. J Clin Oncol. 2005;23(28):7005-7012. doi:10.1200/JCO.2005.01.867

14. Kutikov A, Egleston BL, Wong YN, Uzzo RG. Evaluating overall survival and competing risks of death in patients with localized renal cell carcinoma using a comprehensive nomogram. J Clin Oncol. 2010;28(2):311-317. doi:10.1200/JCO.2009.22.4816

15. Zhang WY, Chen XX, Chen WH, Zhang H, Zou CL. Nomograms for predicting risk of locoregional recurrence and distant metastases for esophageal cancer patients after radical esophagectomy. $B M C$ Cancer. 2018;18(1):879. doi:10.1186/s12885-018-4796-5

16. Chen LL, Nolan ME, Silverstein MJ, et al. The impact of primary tumor size, lymph node status, and other prognostic factors on the risk of cancer death. Cancer. 2009;115(21):5071-5083. doi:10.1002/ cncr.24565

17. Wishart GC, Azzato EM, Greenberg DC, et al. PREDICT: a new UK prognostic model that predicts survival following surgery for invasive breast cancer. Breast Cancer Res. 2010;12(1):R1. doi:10.1186/ bcr2464

18. Kindts I, Laenen A, Peeters S, et al. Validation of the web-based IBTR! 2.0 nomogram to predict for ipsilateral breast tumor recurrence after breast-conserving therapy. Int J Radiat Oncol Biol Phys. 2016;95(5):1477-1484. doi:10.1016/j.jirobp.2016.03.036

19. Jung SP, Hur SM, Lee SK, et al. Validation of a web-based tool to predict the Ipsilateral Breast Tumor Recurrence (IBTR! 2.0) after breast-conserving therapy for Korean patients. $J$ Breast Cancer. 2013;16(1):97-103. doi:10.4048/jbc.2013.16.1.97

20. Shulman LN, Cirrincione CT, Berry DA, et al. Six cycles of doxorubicin and cyclophosphamide or paclitaxel are not superior to four cycles as adjuvant chemotherapy for breast cancer in women with zero to three positive axillary nodes: Cancer and Leukemia Group B 40101. J Clin Oncol. 2012;30(33):4071-4076. doi:10.1200/JCO.2011.40.6405

21. Goldhirsch A, Winer EP, Coates AS, et al. Personalizing the treatment of women with early breast cancer: highlights of the St Gallen international expert consensus on the primary therapy of early breast cancer 2013. Ann Oncol. 2013;24(9):2206-2223. doi:10.1093/annonc/mdt303

22. Zeng D, Zhou R, Yu Y, et al. Gene expression profiles for a prognostic immunoscore in gastric cancer. Br J Surg. 2018;105 (10):1338-1348. doi:10.1002/bjs.10871

23. Jiang $\mathrm{Y}$, Zhang Q, Hu Y, et al. ImmunoScore signature: a prognostic and predictive tool in gastric cancer. Ann Surg. 2018;267(3):504-513. doi:10.1097/SLA.0000000000002116

24. Buchanan CL, Dorn PL, Fey J, et al. Locoregional recurrence after mastectomy: incidence and outcomes. J Am Coll Surg. 2006;203 (4):469-474. doi:10.1016/j.jamcollsurg.2006.06.015

25. Truong PT, Sadek BT, Lesperance MF, et al. Is biological subtype prognostic of locoregional recurrence risk in women with pT1-2N0 breast cancer treated with mastectomy? Int J Radiat Oncol Biol Phys. 2014;88(1):57-64. doi:10.1016/j.ijrobp.2013.09.024

26. Jagsi R, Raad RA, Goldberg S, et al. Locoregional recurrence rates and prognostic factors for failure in node-negative patients treated with mastectomy: implications for postmastectomy radiation. Int J Radiat Oncol Biol Phys. 2005;62(4):1035-1039. doi:10.1016/j. ijrobp.2004.12.014 
27. Truong PT, Lesperance M, Culhaci A, Kader HA, Speers CH, Olivotto IA. Patient subsets with T1-T2, node-negative breast cancer at high locoregional recurrence risk after mastectomy. Int $J$ Radiat Oncol Biol Phys. 2005;62(1):175-182. doi:10.1016/j. ijrobp.2004.09.013

28. Sharma R, Bedrosian I, Lucci A, et al. Present-day locoregional control in patients with $\mathrm{t} 1$ or $\mathrm{t} 2$ breast cancer with 0 and 1 to 3 positive lymph nodes after mastectomy without radiotherapy. Ann Surg Oncol. 2010;17(11):2899-2908. doi:10.1245/s10434-010-1089$\mathrm{x}$
29. Matsubara N, Mukai H, Itoh K, Nagai S. Prognostic impact of Ki-67 overexpression in subgroups categorized according to St. Gallen with early stage breast cancer. Oncology. 2011;81(5-6):345-352. doi:10.1159/000334920

30. Truong PT, Yong CM, Abnousi F, et al. Lymphovascular invasion is associated with reduced locoregional control and survival in women with node-negative breast cancer treated with mastectomy and systemic therapy. J Am Coll Surg. 2005;200(6):912-921. doi:10.1016/j. jamcollsurg.2005.02.010

\section{Publish your work in this journal}

Cancer Management and Research is an international, peer-reviewed open access journal focusing on cancer research and the optimal use of preventative and integrated treatment interventions to achieve improved outcomes, enhanced survival and quality of life for the cancer patient.
The manuscript management system is completely online and includes a very quick and fair peer-review system, which is all easy to use. Visit http://www.dovepress.com/testimonials.php to read real quotes from published authors. 